\title{
Grid technologies for Intelligent Autonomous Robot Swarms
}

\author{
Fabio P. Bonsignorio \\ Heron srl \\ Italy
}

\section{Introduction}

This chapter shows how grid computing characteristics enable the deploying of huge networks of comparatively intelligent robotic agents.

It describes and discusses the concept of a grid based distributed multiagent control architecture for intelligent autonomous robot swarms, in the perspective of network robotics state of the art and the coming pervasive computing and communication scenario.

It underlines the issues and opportunities created by the general objective of harnessing the huge on demand computing power provided by grid technologies to expand the capabilities of networked robotic systems.

As an example it is shown how they are embedded into RobotGrid middleware system.

It implements a planning, control and cooperation architecture which is grid based and exploits the n-tiered java and wireless technologies of common use in the world wide web, see Fig. 1.

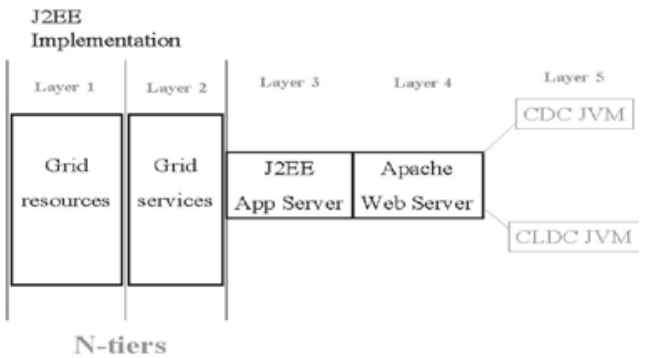

Fig. 1. The n-tiers.

Moreover from a computer science standpoint it is interesting to blend grid and multiagent system technology paradigms.

RobotGrid system architecture is a natural application of common web application design concepts to the task we have identified. The most common application environments which enable this kind of architectures are Java 2 Enterprise Edition (J2EE) and .Net framework. A few hints about the 'enabling' technologies are recalled.

Those technologies are the 'grid' infrastructure itself, a - comparatively - widespread development toolkit for interfacing to grids (the 'Globus' toolkit), the grid service implementation. 
According to various sources (e.g. Gartner Research, the technical report on Pervasive Computing and Communications Thematic Group Report of the IST Beyond the Horizon project), we are moving, from 2006 and beyond, towards an era of pervasive computing and communication devices.

In the meantime the "moore's law" is continuing to work (although in ten/twenty years we could approach the physical limits of VLSI technology), see Fig 2.

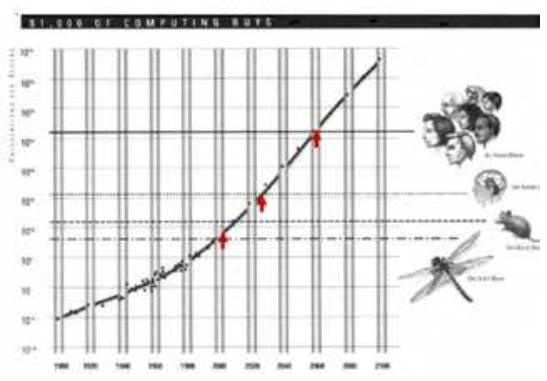

Fig. 2. 1000 dollars buy (from 'The Age of Spiritual Machines', R.Kurzweil).

According to Gartner the next years will see the spreading of:

- $\quad$ pervasive wireless

- $\quad$ real-time infrastructure (grids)

- $\quad$ service-oriented architecture

- low power-consumption mobile devices

There are some visionary statements, like L.V.Gertsner (former IBM's CEO) talking about “...a billion people interacting with a million e-businesses with a trillion intelligent devices interconnected ...".

There are some important international initiative like the South Korean Ministry of Information and Communication URC (Ubiquitous Robotics Companion) project.

Grid computing characteristics enable the deploying of huge networks of comparatively intelligent robotic agents. We describe here a middleware architecture which is grid-based and exploits the n-tiered java and wireless technologies of common use in the world wide web in the first stage of development and a new concept highly distributed swarming autonomous multi robot system as a further step.

As a typical real time multiagent application it constitutes an interesting challenge for the grid paradigm: as a consequence the possible fields of application of grid technologies are expanded making possible new applications in new domains like, for example, interactive multimedia user interface applications.

A grid infrastructure can be regarded as a distributed computing infrastructure which enables the supply of computational capacity on demand.

This kind of infrastructure can deploy very high calculation performance to a great number of points of service with a great variation as a function of time, position, serviced unit.

Actually, this fits the elaboration needs of each individual robotic agent in the considered environments, which can lead to very different computational loads from robot to robot in a given time frame. This allows to optimize the exploitation of the available overall capacity. Self adaptation capabilities are enhanced: for instance, the destruction of a robotic agent can be overcome moving another one in the same place and substituting the 'mind' of the out of order robot to the one substituting it. 


\subsection{Network robotics}

Networked robotics is a new and growing area of research integrating robotics, networking, multimedia and component-based software technologies in support of local, remote, distributed, cooperative and multi-robot system architectures and operations.

Networked robotics constitutes a new conceptual framework in which to explore and extend traditional problems in robotics, while creating important new robotics applications.

A paradigm shift (in the T. Kuhn sense) is emerging: from designing an architecture that integrates sensors and actuators within a single physical platform under centralized control to one in which robotic sensors, actuators, computing, and human interfaces are distributed across multiple physical robot platforms, possibly in time-delayed and/or asynchronous communications.

There are several important research project in this field, some of the most important are the already quoted Korean KIST URC project, US DARPA MARS Vision 2020, Japan Network Robot Forum.

Close in concept are the US ARMY FCS initiative and the DARPA Coabs project.

The URC and the Network Robot Forum motivation are clearly showing, on top the scientifical challenges the economical relevance of the network robotics field. This project in particular looks like an extension to robotics of the thin-client concept of internet computing: 'the network is the robot'.

Network Robot Forum estimates the networked robot industry to grow to 19.8 trillion yen (>130 G€) by 2013.

KIST hope to put a 'network robot' in any Korean household (100\% penetration!) by year 2010 (actually S. Korea has a strong high bandwidth internet penetration.

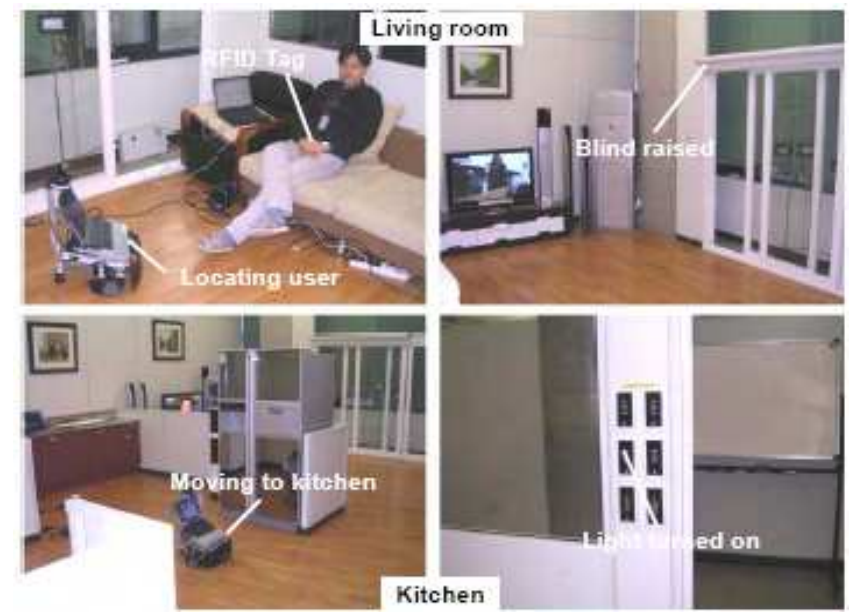

Fig. 3. Examples of Network Robot usage (courtesy KAIST).

There is a clear need for research and ongoing research activities in many fields, spanning from research on sensor, actuator, and processor resources to the development of models for collective sensing and decentralized control in distributed robotic systems and of modular, extensible architectures for multi-robot interaction and real-time coordination

Moreover we can see extensions of the Networked Robotics paradigm and architectures to related problems: smart structures, smart home, pervasive computing and ambient intelligence and to the problem of cognition itself: (multiagent) cognition 
could/should be seen as a cooperative effort raising question spanning from computer science to epistemology, could we talk of a 'network embodied cognition'?

\subsection{Multi Agent Systems and Multi Robot Systems}

The emergence of autonomous agents and multi-agent technology is one of the most exciting and important events to occur in computer science during the 1990s. It is widely believed that this technology will play a central role in the development of complex distributed systems, networked information systems, and computer interfaces during the next decades.

Multi Robot Systems (MRS) are a particular form of multi agent system.

There is a natural exchange of concepts and methods between the two areas.

The advantage of a MRS are in term of the effectiveness and performance and in term of robustness and reliability (Dudek, 1996; Parker, 1998). MRS research and development is affected by recent research on biological systems and by new models in cognitive science and economics (Cao, 1997), sometimes influenced by complex system theory. According to the number and kind of mutual interaction between the robotic agent of the multi robot system can be regarded as a swarm (Cao, 1997; Parker, 1998), or a colony (Arkin, 1997) or, more generally, as a robot collective (Dudek et al., 1996).

A special kind of MRS are the RoboCup teams (Asada, 1998; Kitano et al., 1999). An interesting feature of RoboCup and similar environments, is that the RoboCup environment is not only highly dynamic but also involve an opponent robotic network.

\subsection{Grid and Multi Agent systems}

There is a common underlying thread between multiagent and grid technologies, the creation of networks of entities (call them communities or VO) with a common goal.

In the case of grids the focus is on how the communities form and operate, on the other hand, the understanding of how this can be used in order to obtain large scale systems with stable collective behaviour is less mature.

Commonly available Grid tools provide mechanisms for accessing data on different storage system but not for the semantic integration of that data.

Grids need flexible decentralised decision capabilities agents need a robust distributed computing platform allowing them to discover acquire federate and manage the capabilities necessary to execute their decisions.

One approcah is layering the two technologies i.e. put a multiagent system on top of a grid infrastructure.

It seems likely that a grid/agent approach will give better results with a fine grain interwining of the two technologies.

\subsection{Pervasive computation scenario}

The large scale computing, grid, paradigm shift is enabled by recent advances in computer science, with parallel and distributed computing playing a key role.

The grid paradigm offers an important abstraction for combining national and continent wide computational resources..

Driven by the increase in internet usage grid computing will provide the next phase in the use and dissemination of knowledge.

The grid can be seen as a way for integrating resources which can span from dedicated high performance arrays of computers to low end clusters of workstation. 
To manage this complexity a kind of AI behaviour is needed.

\section{RobotGrid Architecture}

\subsection{Grid Infrastructure}

Grid technologies support the sharing and coordinated use of heterogeneous resources (memory, cpus, devices of any kind) in dynamic computing network environments. This networks of resources can be distributed on a local area network, or geographically on an intranet but also over several (virtual) organisations or the WWW.

The key distinctive point of this kind of infrastructures is that resources are not statically allocated to a specific service / task (or pool of services)but they are assigned dynamically on the basis of current needs.

Thus allowing an optimal exploitation of available resources.

An other remarkable advantage, which could be of great help in deploying large scale or intelligence intensive application of the kind we are considering here, connecting to a public large scale grid could provide unprecedented high performance computing capabilities enabling a whole array of advanced highly effective applications.

Grid technologies were conceived in order to support scientific cooperation on various issues from (Catlett, 1992; Catlett et al., 1992; Foster, 2002; Foster \& Kesselman, 1999a; Johnston et al., 1999; Stevens et al., 1997), graphical rendering of large databases, high computation needs for data analysis, integration of scientific instruments with remote data and computing resources (Johnston, 1999).

It is reasonable to envision applications of this kind of technologies to the robotic field where the management of dynamic sets of resources and services can be of great help.

In practice it is necessary a set of 'API' (Application Programming Interface' in order to be able to really deploy a given software system on a given platform

The Globus toolkit is one example of this set of API.

\subsection{The Globus Toolkit}

The Globus Toolkit (Foster \& Kesselman, 1999b; Foster et al., 2001) is a community-based, open-architecture, open-source set of services and software libraries that support Grids and Grid applications. The Globus Toolkit has been adopted by hundreds of sites and by many of the chief Grid projects around the world.

Globus includes a set of basic utilities:

- Grid Resource Allocation and Management (GRAM) protocol which supplies service creation and management

- Meta Directory Service (MDS-2), which supplies information discovery

- Grid Security Infrastructure (GSI), which supports single sign on, delegation and other security features

From our standpoint this kind of toolkits are the ground base for implementing web services which make available to the planning and control system grid functions in order to exploit the expected computation power advantages.

\section{3 'Grid services'}

A 'grid service' is a web service that provides a set of grid functions.

A key point are transient service instances. Serviced units typically do not require a set of 
persistent services for handling their needs, but, instead, they need 'transient' service instances to manage a particular time limited state of an ongoing activity, when the activity finishes the service is no longer needed and the instance can be destroyed.

In our case service instances could be instantiated dynamically to supply dynamically supplemental computing, memory and bandwidth resources in order to cope with a transient workload.

\subsection{RobotGrid tiered structure}

As told above, the middleware architecture described here is a natural application of common web application design concepts to the task of enabling innovative multi robot system applications.

RobotGrid middleware architecture is based on J2EE.

Within the research community is emerging a set of 'new generation' web technologies which are collective known as 'grid' technologies. A bunch of products exploiting various aspects of this methods are already available on the market (e.g. Oracle 10g Application Server).

We will see below how this methods can be blended in order to achieve an environment suitable for advanced robotic applications.

And how the overall capabilities of a 'robot grid' can be augmented interfacing a one or more wireless sensor networks. In order to better explain the suggested solution, a few hints about the 'enabling' technologies are recalled below.

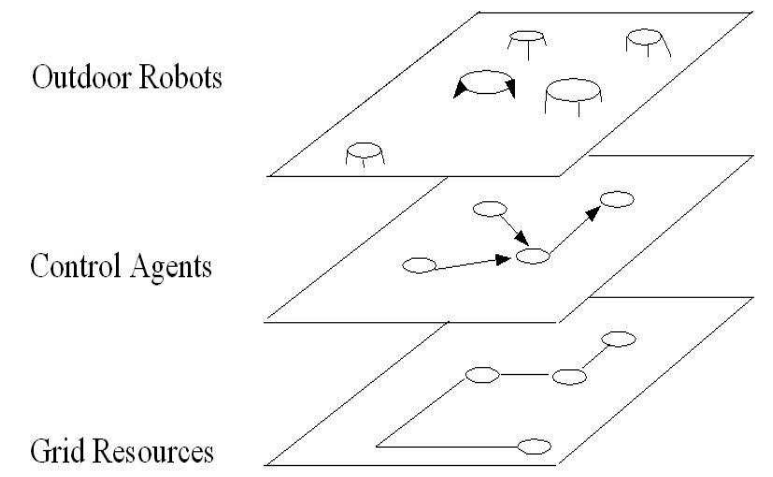

Fig. 4. RobotGrid layered architecture.

Those technologies are the 'grid' infrastructure itself, a - comparatively - widespread development toolkit for interfacing to grids (the 'Globus' toolkit), the grid service implementation, the WSN technologies.

The n-tiered structure of the RobotGrid middleware is depicted in Fig. 5. At the lower layer we have the infrastructure resources: computers with their operating systems, san (storage area network), nas (network area system), (remote)sensors,(remote) robots. Immediately above we have the grid services layer, which implements the virtualization of the resource of the bottom level, according to the grid service paradigm on demand. Then we have the RTRDBMS (Real Time Relational Data Base System), RTKBMS (Real Time Knowledge Base Management System) and other data and rule application management system, simulation tools. Above we have the application server level, where the (e.g. enterprise java) beans implementing the 
planning and control logic reside. The DB and Application are here together regarded as the 'Layer 3'.

On this layer there are beans calling from their code for instance the Globus APIs, other interfacing for instance the Java Virtual Machine embedded in the autonomous robot, other implementing the Planning and Control Logic.

The web serving layer, which make available through the http protocol the complex services made possible by the overall architecture.

The onboard virtual machine, which is the part of the system that necessarily resides on the robot and manages by means of 'applets' the physical interfaces of the robot and the ones with the control algorithms, rules and methods. A description of an application deployment environment fit for this use (Java 2 Micro Edition) in described in the next paragraph.

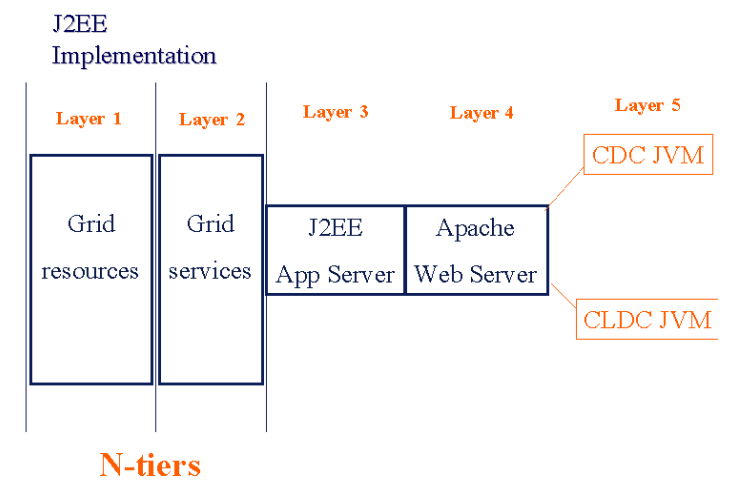

Fig. 5. The n-tiers.

We show in figure 6 the flow of the sense/reaction scheme of a single robot.

The 'applet' running on the robot onboard virtual machine sense e.g. a movement of a 'focus object' in the 'environment'.

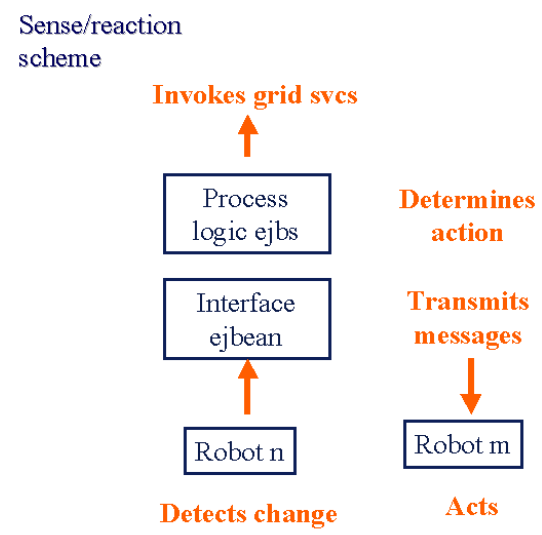

Fig. 6. The sense/reaction scheme. 
A front end unit of service (an interface 'enterprise java bean') is invoked with a string of parameters, the bean activate the necessary actions instantiating the necessary transient grid services (adding resources where necessary) and some where another bean dispatch a message that trough an on board applet act in the environment (e.g. moving one or more robot on the field from one position to another) .

From a simplified standpoint, we can say each robot in the 'swarm' is represented by a software agent within RobotGrid.

Immediately above we have the grid services layer, which implements the virtualization of the resource of the bottom level, according to the grid service paradigm on demand.

This layer is running on a soap server and wraps the underlying grid services implemented encapsulating of one of the grid service sets under development by various organization or, directly, the java cog apis.

The exposed web services are invoked by the set of ejbeans running within a J2EE application server container mapping the swarm as a collection of RobotJbeans and Stateful Session Beans representing the individual robot of the physical swarm.

A very simplified schema is depicted in fig. 7.

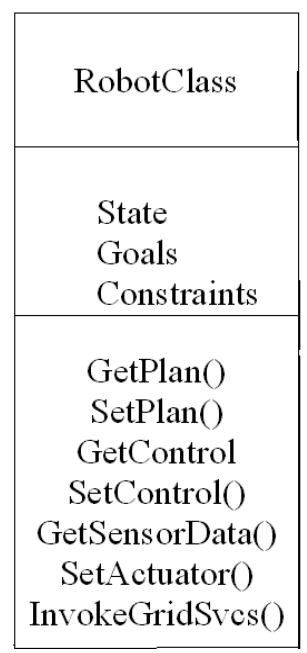

Fig. 7. The Robot Class

Within the same (logical) application server run the wsn set of wsn ejbeans mapping the sensor network wrapping the call to the wsn subnetworks gateways, for instance Intel/Crossbow Stargate devices.

A schematic is shown in fig. 8 .

\section{Issues and opportunities}

The architecture described here is largely based on 'commodity' software environments . There are, although, limits that prevent from some extreme application.

For example the smaller robotic unit should - without workarounds and with the constraint of using commodity system environment like those quoted above - be in the $10 \mathrm{~cm}$ scale. 
Fig. 8. The Wsn Class.

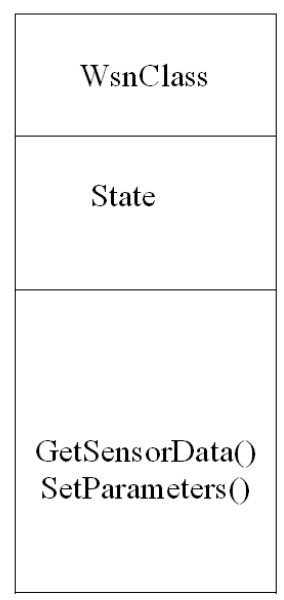

The environment is complex and although capable of supplying high computing power it requires some resources.

The biggest issues regard Quality of Service, this kind of application requires:

- Execution time predictability

- $\quad$ Predictable scheduling algorithms;

- $\quad$ Predictable cost models for parallel algorithms;

- $\quad$ Failure handling and graceful degradation on the Grid side

This can be achieved by means of scheduling algorithms, exploiting:

- $\quad$ Staging of executable files and data;

- Application start-up and overhead reduction and predictability;

- Advanced allocation policies;

- $\quad$ Resource Agreement reliability;

On the other end, the real strength of the proposed architecture is that an inherently on demand flexible and distributed grid based computing environment is coupled with a distributed sensing/actuating environment.

Another advantage is the capability to allocate the computing resources to the robot units which really need them when and how they need them,

Given that and considering that the physical implementation and 'low' level control algorithms perform in parallel as well as the robot tasks and goals and the most common computing tasks (planning for all) are of a kind that can greatly benefit from parallel execution with are best condition to fully exploit advantages of the underlying architecture.

Last but not least the computing grid can be a disconnected local one, but also a big Virtual Organisation grid or even 'the Grid', as a consequence the deployed computing power can be quite noticeable.

Today, applications of networked automation are already pervasive in most fields of human endeavor. The networked automation tools and technologies have a wide range of applications. New questions, that could not have been formulated a few years ago, are being posed, mainly due to the technological advancements in robotics, control, computing and communication. Again, these technological developments made 
possible to envision the implementation of systems which could have not been imagined before.

Network and networked automation are already ubiquitous and pervasive in most areas of daily experience of citizens in the old and new indutrialised countries.

The new advances in robotics (in particular service robotics), control (in particular distributed control), computing and communications (in particular the web, the semantic web, the grid, object of this proposal), create the opportunities for new product, services, new 'service-products'.

As a consequence of this technological driving force, the transversal field on network robotics is gaining momentum enabling a broad range of new solutions for old problems and new opportunities.

- multi-vehicle systems, robotized highways, waterways

- domotics

- Industrial automation: wireless automation and wireless factory; new generation robots, multi agent systems, holonics systems

- Ambient intelligence and intelligent spaces

- Health care: sensor networks for monitoring and intervention; integrated robotic help;

- remote interventions; automated drug delivery, etc.

- Earth sciences: networks of sensors and robotic devices for detailed scientific field

- studies in remote and hostile environments inaccessible to humans; studies of global

- warming, etc.

- Disaster management: ; USAR, intervention in disaster

- scenarios; fire detection, management and fire fighting, etc.

- Security, survelliance and civil defense: deployment of interoperated sensor and communication

- networks in disaster scenarios; surveillance and security

- Agriculture: crop monitoring and interventions; optimization of irrigation systems; harvesting

- Forest management

- Constructions

- Urban maintenance

- humanitarian demining

- mining operations

- Management of natural resources: networks of sensors and robotic vehicles for water management; air quality; etc.

- Military: new strategic concepts and tactical deployments rely heavily on network centric concepts, which involve not only manned systems but with an increasing rate on sensor and robotic vehicle networks (US Army Future Combat System)

- An important aspect of networked robotics system is their 'multi role' characteristics a middleware for network robotics can in principle be used for USAR or for urban cleaning or for forest management.

Making the grid technologies available for network robotics means to set up an enabling technological platform which could prepare the advent of a (huge) number of 
new service products ready for the upcoming pervasive computing and communication scenario.

\section{Reliability and Performance}

The RobotGrid grid environment is a quite complex one, as a consequence giving quantitative prediction of a network robot system based on this middleware can be difficult, nevertheless it is possible to draw some conclusions on the basis of simple assumptions, on the basis of system reliability theory and of multi server system performance evaluation techniques.

Since it is not possible to define an explicit closed form model of the sytem, better predictions can be obtained by simulation and this is part of ongoing research.

\subsection{Modeling Reliability}

The reliability of a system is the probability that it is working properly in an given time period.

The reliability of a system made of $\mathrm{n}$ parellel component is given by:

$$
R_{p}=1-P_{r}
$$

where, $r_{i}$ reliability of the $i$ component:

$$
P_{r}=\prod_{i=0}^{n-1}\left(1-r_{i}\right)
$$

As a consequence:

$$
R_{p}=1-\prod_{i=0}^{n-1}\left(1-r_{i}\right)
$$

if $r_{i}=r=$ const, we have:

$$
R_{p}=1-(1-r) n
$$

In Fig. 9 we see how with several values of $r$ we get a very high level of reliability with a small number of parallel components: in our case both the robots in the physical world and the computing resources supplied by the grid are sets of parallel resources.

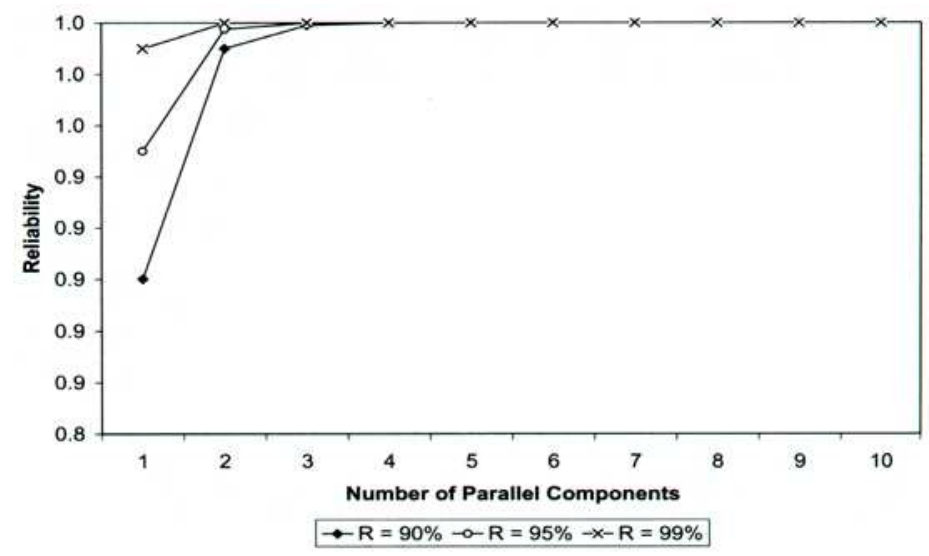

Fig. 9. Reliability of parallel components. 


\subsection{Modeling Performance}

Let $\lambda$ (request/s) the average arrival rate of rate of requests, $\mu$ (request/s) the average request throughput of the servers.

Assuming infinite population and finite queue, we get,

$P_{k}$ the fraction of time the server has $\mathrm{k}$ request

$$
p_{k}= \begin{cases}\frac{1-\lambda / \mu}{1-(\lambda / \mu)^{W+1}}\left(\frac{\lambda}{\mu}\right)^{k} & k=0, \ldots, W \quad \lambda \neq \mu \\ 1 /(W+1) & k=0, \ldots, W \quad \lambda=\mu\end{cases}
$$

U, server utilization:

$$
U= \begin{cases}\frac{(\lambda / \mu)\left[1-(\lambda / \mu)^{W}\right]}{1-(\lambda / \mu)^{W+1}} & \lambda \neq \mu \\ W /(W+1) & \lambda=\mu\end{cases}
$$

$\mathrm{X}$, average server throughput:

$$
X=U \times \mu
$$

$\bar{N}$, average number of requests in the server:

$$
\bar{N}= \begin{cases}\frac{(\lambda / \mu)\left[W(\lambda / \mu)^{W+1}-(W+1)(\lambda / \mu)^{W}+1\right]}{\left[1-(\lambda / \mu)^{W+1}\right](1-\lambda / \mu)} & \lambda \neq \mu \\ W / 2 & \lambda=\mu\end{cases}
$$

The previous equations lead to:

$$
R=\bar{N} / X
$$

In Fig. 10 is plotted the average response time $(R)$ of an infinite population/finite queue multiserver system, according to equation (9).

It shows how there is a threshold number of servers (in the ideal model) above which the response time can be made very low.

This suggests the usefulness of adding computing capacity on demand.

The plot assumes 500 requests/s from the 'swarm', a limit of 200 simultaneous connection and of 20 request/s for each identical server.

\section{System Simulation}

As told above, since it is not possible to define an explicit closed form model of the system, when designing a solution for a specific application the behaviour of the system should be supported by a simulation tool. 


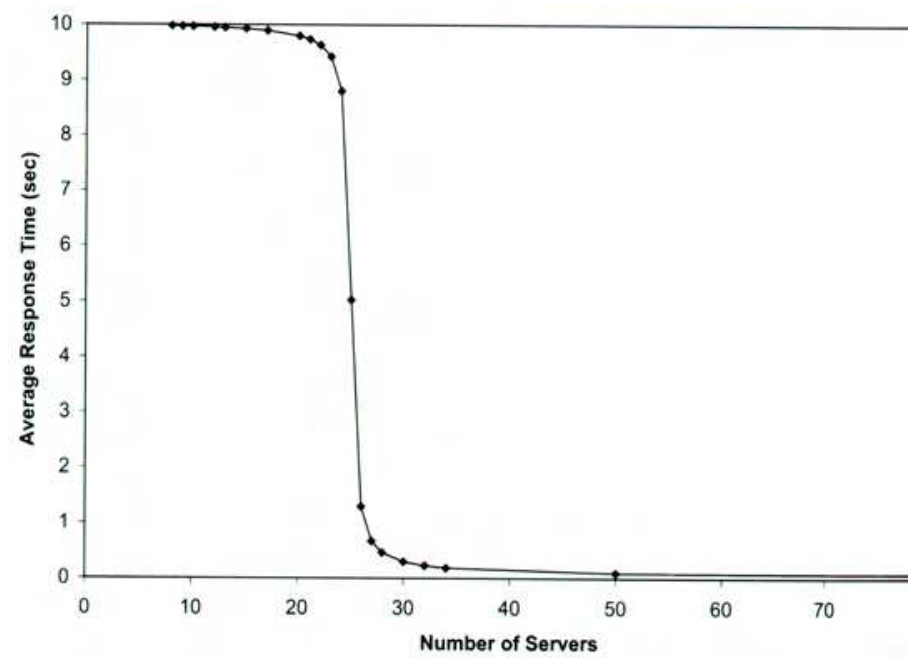

Fig. 10. Response time vs. number of servers.

The simulation of the system is performed in Webots (Webots, 2006).

The multirobot system is constituted by a variable number of Khepera robots (see Fig. 11).

Khepera is a mini mobile robot produced by K-team SA, Switzerland.

It is a caster-like robot with two differential wheels, which can be equipped with various kind of sensors and actuators.

In our first simulations the Khepera are identical and are equipped with 7 distance sensor and 7 light sensors.

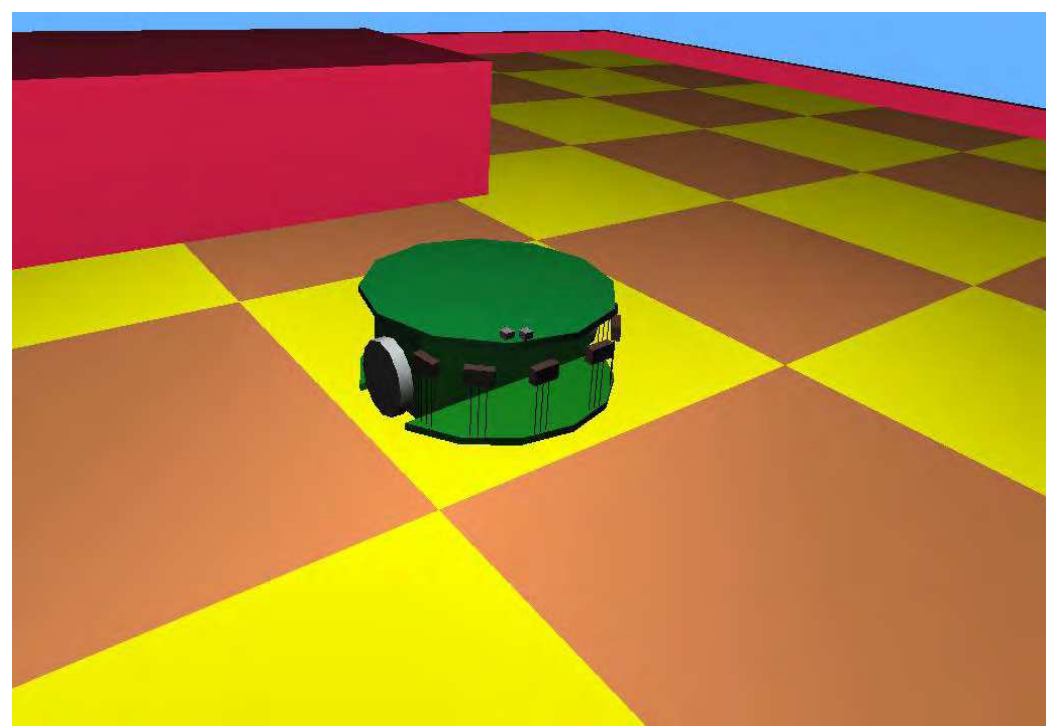

Fig. 11. The Khepera robot. 
The 'environment' where they are tested is very simple, see Fig. 12.

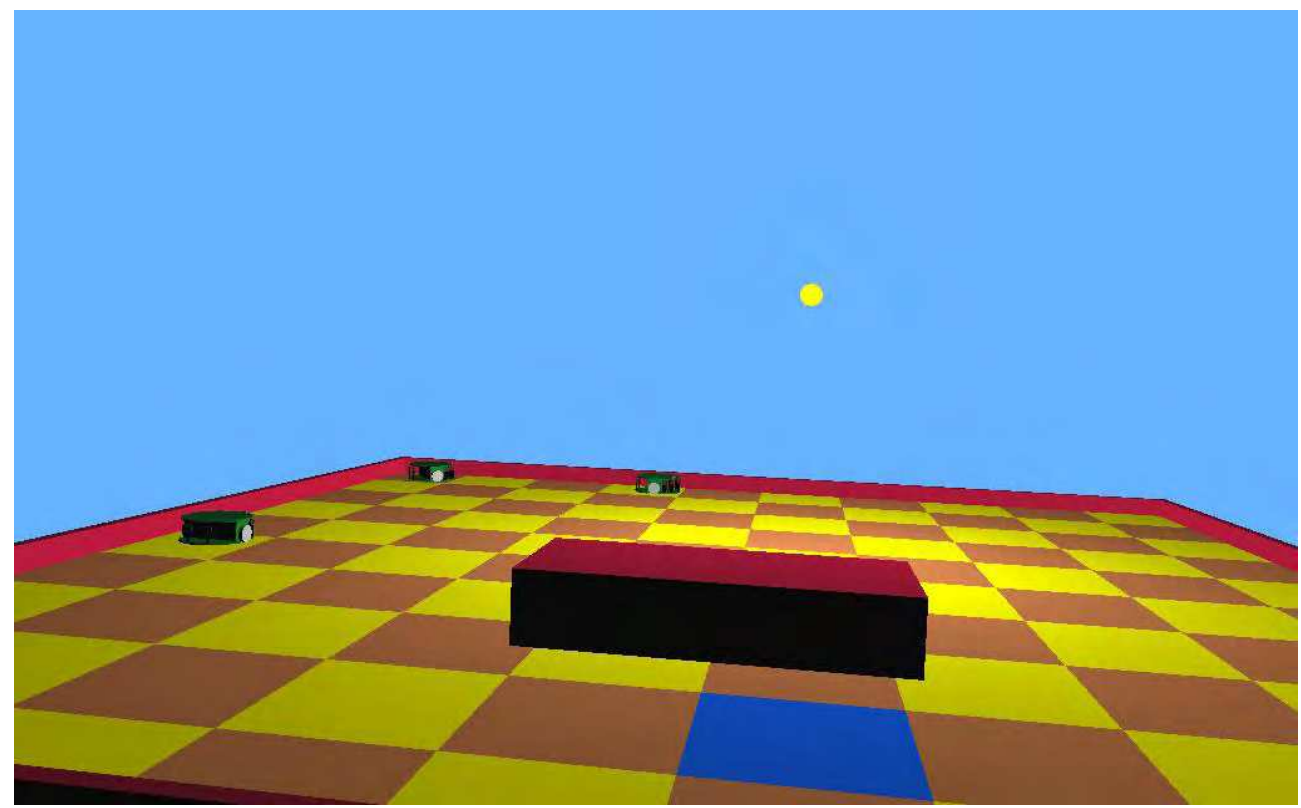

Fig. 12. The simulated environment.

In the future we will test more diversified 'swarms' in environments made progressively more complex.

The Khepera robots can be controlled remotely through a tcp/ip interface. They are suitable to be interfaced to the architecture we have described.

The grid infrastructure is simulated as a variable set of computing and communication resources.

\section{Further Research}

We have described at a conceptual level the architecture of a middleware system for intelligent robot swarms integrated with one or more wireless sensor networks.

It is ongoing the simulation and implementation of the software architecture in specific cases and the physical implementation in some relevant case among those quoted above.

Possible application examples could be humanitarian demining, robotic cleaning 'agency' and intelligent vehicles for urban and suburban mobility.

\section{Conclusions}

From a certain respect what is described above can be seen as a generalized grid where some of the resource managed are various kind of intelligent autonomous robotic agents.

This seems to show some inherent advantages. 
If the computing grid, is a VO grid, or 'the grid' the high performance computing capabilities added to the multiplication effect in the 'real world' of deploying a swarm of intelligent autonomous robots could apparently open a wide range of new advanced applications.

Moreover, from a computer science standpoint it is interesting to blend a multiagent system with a grid infrastructure, while the Quality of Service requirements of a real time system expand the reach of application of grid technologies.

As we move towards a pervasive computation and communication scenario, where grid computing facilities will (or could) become a commodity service, the kind of architecture we described here, could become quite common.

\section{References}

Arkin, R.C. \& Bekey, G.A. Robot Colonies (1997) Kluwer Academic Publishers, 1997.

Asada, M. (1998) The RoboCup physical agent challenge: Goals and protocols for Phase-I. In Kitano, H. editor, RoboCup-97: Robot Soccer World Cup I, 1998.

Cao, Y. ; Fukunaga, A. \& Kahng, A. (1997) Cooperative mobile robotics: Antecedents and directions. Autonomous Robots, 4:1-23, 1997.

Catlett, C. (1992) In Search of Gigabit Applications, IEEE Communications Magazine (April).42-51. 1992.

Catlett, C. \& Smarr, L. (1992) Metacomputing, Communications of the ACM, 35 (6). 44--52. 1992.

Dudek, D. ; Jenkin, M. ; Milios, E. \& Wilkes, D. (1996) A taxonomy for multi-agent robotics. Autonomous Robots, 3(4):375-397, 1996.

Foster, I. (2002) The Grid: A New Infrastructure for 21st Century Science, Physics Today, 55 (2). 42-47. 2002.

Foster, I. \& Kesselman, C. (1999) Globus: A Toolkit-Based Grid Architecture, In Foster, I. And Kesselman, C. eds. The Grid: Blueprint for a New Computing Infrastructure, Morgan Kaufmann, 1999, 259-278.

Foster, I. \& Kesselman C. (eds.). (1999) The Grid: Blueprint for a New Computing Infrastructure Morgan Kaufmann, 1999.

Foster, I.; Kesselman, C. \& Tuecke, S. (2001) The Anatomy of the Grid: Enabling Scalable Virtual Organizations, International Journal of High Performance Computing Applications, 15 (3). 200-222, 2001.

Johnston, W. (1999) "Realtime Widely Distributed Instrumentation Systems" In Foster, I. and

Kesselman, C. (1999) In Foster, I. And Kesselman, C. eds. The Grid: Blueprint for a New Computing Infrastructure, Morgan Kaufmann, 1999, 75-103.

Johnston, W.E.; Gannon, D. \& Nitzberg, B. (1999) “Grids as Production Computing Environments: The Engineering Aspects of NASA's Information Power Grid. " In Proc.8th IEEE Symposium on High Performance Distributed Computing, (1999), IEEE Press

Kitano, H. , Pagello, E. \& Veloso, M. (1999) editors RoboCup-99: Robot Soccer World Cup III, Springer-Verlag, 1999.

Parker, L.E. (1998) ALLIANCE: An architecture for fault tolerant multirobot cooperation, IEEE Transactions on Robotics and Automation, 14(2):220-240, 1998. 
Stevens, R.; Woodward, P.; DeFanti, T. \& Catlett, C. (1997) From the I-WAY to the National Technology Grid, Communications of the ACM, 40 (11). 50-61. 1997.

Webots (2006) http://www.cyberbotics.com. Commercial Mobile Robot Simulation Software 


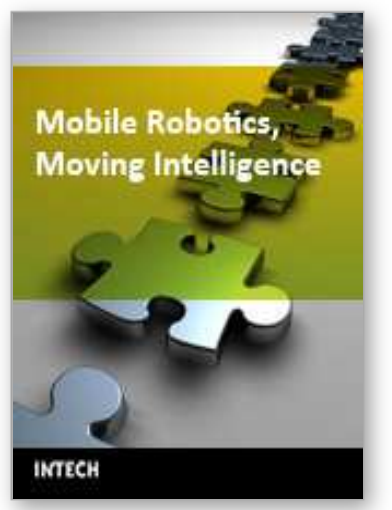

\author{
Mobile Robotics, Moving Intelligence \\ Edited by Jonas Buchli
}

ISBN 3-86611-284-X

Hard cover, 586 pages

Publisher Pro Literatur Verlag, Germany / ARS, Austria

Published online 01, December, 2006

Published in print edition December, 2006

This book covers many aspects of the exciting research in mobile robotics. It deals with different aspects of the control problem, especially also under uncertainty and faults. Mechanical design issues are discussed along with new sensor and actuator concepts. Games like soccer are a good example which comprise many of the aforementioned challenges in a single comprehensive and in the same time entertaining framework. Thus, the book comprises contributions dealing with aspects of the Robotcup competition. The reader will get a feel how the problems cover virtually all engineering disciplines ranging from theoretical research to very application specific work. In addition interesting problems for physics and mathematics arises out of such research. We hope this book will be an inspiring source of knowledge and ideas, stimulating further research in this exciting field. The promises and possible benefits of such efforts are manifold, they range from new transportation systems, intelligent cars to flexible assistants in factories and construction sites, over service robot which assist and support us in daily live, all the way to the possibility for efficient help for impaired and advances in prosthetics.

\title{
How to reference
}

In order to correctly reference this scholarly work, feel free to copy and paste the following:

Fabio P. Bonsignorio (2006). Grid Technologies for Intelligent Autonomous Robot Swarms, Mobile Robotics, Moving Intelligence, Jonas Buchli (Ed.), ISBN: 3-86611-284-X, InTech, Available from:

http://www.intechopen.com/books/mobile_robotics_moving_intelligence/grid_technologies_for_intelligent_auto nomous_robot_swarms

\section{INTECH}

open science | open minds

\section{InTech Europe}

University Campus STeP Ri

Slavka Krautzeka 83/A

51000 Rijeka, Croatia

Phone: +385 (51) 770447

Fax: +385 (51) 686166

www.intechopen.com

\section{InTech China}

Unit 405, Office Block, Hotel Equatorial Shanghai

No.65, Yan An Road (West), Shanghai, 200040, China 中国上海市延安西路65号上海国际贵都大饭店办公楼405单元

Phone: +86-21-62489820

Fax: +86-21-62489821 
(C) 2006 The Author(s). Licensee IntechOpen. This chapter is distributed under the terms of the Creative Commons Attribution-NonCommercialShareAlike-3.0 License, which permits use, distribution and reproduction for non-commercial purposes, provided the original is properly cited and derivative works building on this content are distributed under the same license. 